\title{
Maltraitance envers les enfants déclarée par les jeunes eux-mêmes à l'occasion d'enquêtes représentatives de la population : revue systématique
}

Jessica Laurin*, M.A.; Caroline Wallace*, B. Sc.; Jasminka Draca, B. Sc. S.; Sarah Aterman, B.A.S.; Lil Tonmyr, Ph. D.

Cette synthèse des données probantes a fait l'objet d'une évaluation par les pairs.

Diffuser cet article sur Twitter

\section{Résumé}

Introduction : Cette revue systématique a permis de recenser des enquêtes sur les jeunes représentatives de la population et comportant des questions sur la maltraitance envers les enfants déclarée par ces derniers. Nous avons également examiné la qualité des données et les questions d'ordre éthique pertinentes pour la collecte de données sur la maltraitance.

Méthodologie : Nous avons effectué une recherche dans diverses bases de données en ligne pour sélectionner les articles publiés entre janvier 2000 et mars 2016 qui contenaient des données représentatives de la population mesurant la maltraitance envers les enfants. Les critères d'inclusion ont été établis a priori et deux examinateurs ont évalué indépendamment l'un de l'autre les articles pour s'assurer que les critères étaient respectés et pour vérifier l'exactitude des données extraites.

Résultats : Au total, 73 articles portant sur 71 enquêtes ont répondu aux critères d'inclusion. Nous avons relevé diverses stratégies visant à assurer l'exactitude des renseignements et à atténuer la détresse des participants à l'enquête.

Conclusion : L'ampleur des efforts déployés pour mesurer la prévalence de la maltraitance envers les enfants est le reflet de l'importance accordée à cette dernière à l'échelle de la planète. Malgré des connaissances incomplètes en matière de pratiques exemplaires liées à l'éthique et de qualité des données, il est possible de recueillir efficacement auprès des jeunes eux-mêmes des données sur la maltraitance dont ils font l'objet.

Mots-clés : abus, négligence, violence, qualité des données, éthique, adolescence, adolescent, revue systématique

\section{Introduction}

Les conséquences de la maltraitance envers les enfants - un enjeu de santé publique particulièrement difficile à quantifier et à étudier — s'étendent bien au-delà du préjudice immédiat infligé. Il a par exemple été démontré que des antécédents de maltraitance envers un enfant nuisent à son développement à l'adolescence et augmentent le risque de certaines des principales causes de morbidité et de mortalité ${ }^{\text {, en }}$ particulier les blessures liées à l'alcool, la consommation de drogues, les comportements d'automutilation, le suicide et l'exposition à la violence ${ }^{2-5}$.
Un nombre croissant de travaux de recherche visent à estimer l'ampleur du phénomène de la maltraitance envers les enfants et à comprendre la dynamique et le mécanisme de son association avec les résultats en santé ${ }^{6}$. Les enquêtes représentatives de la population offrent la possibilité de quantifier la prévalence de la maltraitance et d'évaluer ses risques en lien avec d'autres conditions sanitaires ou sociales. Bien sûr, dans les enquêtes qui englobent un large éventail de contenu sur la santé, les contraintes d'espace et les intérêts divergents constituent un défi quant à l'inclusion de mesures de la maltraitance envers les enfants. Cependant, la

\section{Points saillants}

- Il est possible de recueillir de façon responsable et éthique auprès des jeunes des données sur la maltraitance des enfants tout en protégeant leur santé et leur bien-être.

- Les jeunes ont rarement fait état de préoccupations quant au fait de répondre à des questions sur la maltraitance dans les enquêtes d'auto-évaluation.

- Lors de nos recherches dans les bases de données, nous n'avons trouvé aucune enquête d'autoévaluation menée auprès de jeunes Canadiens représentative à l'échelle nationale et contenant des variables sur la maltraitance des enfants.

- À l'heure actuelle, il existe peu de mesures autodéclarées fiables et valides de la maltraitance envers les enfants.

contribution potentielle de telles enquêtes à l'amélioration de notre compréhension de la prévalence, des facteurs de risque et des répercussions de la maltraitance envers les enfants est de plus en plus reconnue, tant au Canada que dans le reste du monde? Les données de population provenant d'autres pays fournissent une base de comparaisons internationales à partir desquelles nous pouvons examiner l'influence des pratiques culturelles, sociales et stratégiques sur les différences observées ${ }^{8,9}$.

Les aspects éthiques de la recherche sur les enquêtes portant sur la maltraitance envers les enfants sont essentiels. La nature délicate du sujet et le risque de détresse émotionnelle susceptible de s'emparer des 
répondants exigent des mesures de protection de la confidentialité, une administration des questions avec la sensibilité appropriée, l'obtention d'un consentement éclairé et la possibilité d'offrir une interventions de suivi ${ }^{10}$. Les procédures conçues pour aborder ces sujets devraient être clairement définies et faire partie intégrante de toute enquête ou de tout rapport de recherche.

La qualité des données doit être évaluée dans toute recherche par enquête sur la maltraitance envers les enfants. Divers facteurs ont une influence sur la qualité des renseignements fournis par un répondant, en particulier son âge et son stade de développement. L'avantage d'enquêter auprès de jeunes sur l'expérience de la maltraitance est que l'exposition est relativement récente : le biais de rappel est vraisemblablement plus faible que dans le cadre d'une enquête effectuée auprès d'adultes. Les renseignements fournis par les adolescents sont plus fiables que ceux recueillis auprès d'enfants plus jeunes, en raison du développement cognitif plus avancé des premiers ${ }^{11}$. Les recherches semblent ainsi indiquer que les enfants de moins de 10 ans ne sont pas des répondants fiables pour une enquête sur les expériences de maltraitance ${ }^{12}$. La détresse, la gêne et l'embarras générés par la mémoire des événements constituent d'autres obstacles éventuels à la fourniture de renseignements exacts ${ }^{13-16}$.

Un article de synthèse publié en 2000 a porté sur les considérations d'ordre méthodologique et éthique quant au fait d'interroger des enfants sur leur exposition à l'abus physique et sexuel ${ }^{17}$. Les auteurs ont recensé 14 études d'auto-évaluation ayant permis de recueillir des renseignements directement auprès d'enfants, avec des approches très variées pour obtenir ces renseignements ${ }^{17}$. Même si cet examen fournit des données très utiles, il est limité aux enquêtes menées avant 1999, et les enquêtes recensées portent surtout sur l'abus physique et sexuel et ne sont pas représentatives de la population en général. Les auteurs ont noté des écarts considérables dans les méthodes de collecte de données, dans le libellé et le nombre de questions sur la maltraitance ainsi que dans les procédures de consentement. Cela explique que les estimations de l'abus physique et sexuel variaient considérablement.
Notre revue systématique a pour but de mieux comprendre les données sur la maltraitance envers les enfants saisies lors d'enquêtes faisant appel à l'autodéclaration par les jeunes. Nos objectifs spécifiques sont : 1) recenser les enquêtes représentatives dans lesquelles on a recueilli auprès des jeunes des données sur la maltraitance envers les enfants et les facteurs ayant une incidence sur la prévalence (il ne s'agit donc pas d'échantillons cliniques), 2) évaluer la qualité des méthodes employées pour mesurer la maltraitance envers les enfants et 3) évaluer les pratiques et les procédures en matière de traitement des questions d'ordre éthique.

\section{Méthodologie}

Cette revue systématique a été réalisée conformément aux lignes directrices PRISMA ${ }^{18}$, et son protocole est accessible sur demande auprès de l'auteur-ressource.

\section{Recension (stratégie de recherche)}

Nous avons effectué une recherche d'articles évalués par les pairs publiés entre janvier 2000 et mars 2016 dans les bases de données en ligne Embase, Medline, PsycINFO, Global Health, Social Policy and Practice, ERIC, Social Services Abstracts, Sociological Abstracts et ProQuest Public Health. Nous avons utilisé les mots-clés suivants : youth, adolescent, young adult, child, abuse, maltreatment, violence, neglect, assault, rape, representative, national, et school surveys [jeune, adolescent, jeune adulte, enfant, abus, maltraitance, violence, négligence, agression, viol, représentatif, national et enquêtes réalisées dans les écoles]. Les chaînes de recherche employées sont disponibles sur demande auprès de l'auteur-ressource. En outre, nous avons examiné les listes de références des articles retenus pour trouver d'autres articles à inclure potentiellement et nous avons consulté divers spécialistes.

Les critères d'inclusion des articles dans la revue sont :

- articles publiés en anglais

- étude primaire (ni synthèse, ni éditorial)

- données recueillies après 1999

- sources de données restreintes aux enquêtes réalisées dans les écoles ou aux enquêtes représentatives de la population (décrites comme représentatives par les auteurs des articles ou échantillonnées et pondérées afin de refléter précisément les membres de la population dans son ensemble)

- modèle d'étude transversale

- fourchette d'âge des répondants de 10 à 18 ans (groupe d'âge principal), parfois élargie jusqu'à 24 ans

- victimes âgées de moins de 18 ans au moment de l'exposition à la maltraitance

- l'auteur de la maltraitance signalée est un parent ou une personne s'occupant de l'enfant (sauf pour les cas d'abus sexuel, où l'agresseur peut être n'importe qui, mais les articles mettant l'accent sur les pairs ou la victimisation en ligne n’ont pas été retenus)

- analyse effectuée sur l'ensemble de l'échantillon du groupe d'âge sélectionné (10 à 18 ans).

Il est à noter que nous avons limité l'inclusion aux études transversales afin d'assurer l'inclusion du plus grand nombre d'enquêtes. En outre, puisque l'objectif premier de cette revue n'est pas d'établir des associations mais de déterminer s'il est possible de recueillir des données sur la maltraitance auprès de jeunes en vue d'estimer la prévalence, les études transversales sont appropriées. L'avantage d'inclure des études longitudinales serait limité, étant donné que les questions sur la maltraitance envers les enfants sont rarement posées au cours de la première vague d'une étude longitudinale, mais plutôt dans les dernières vagues lorsque l'attrition peut être un problème $e^{19,20}$.

\section{Présélection/admissibilité (processus de sélection)}

La figure 1 illustre le processus de sélection des articles retenus. La recherche dans les bases de données a permis de repérer 4383 articles, puis la consultation d'experts et la recherche dans les références bibliographiques ont permis d'ajouter 31 articles. L'élimination des recherches citées en double a réduit le nombre d'articles à 3885 et, après un tri fondé sur le titre et le résumé, il nous est resté 220 articles à évaluer. Deux examinateurs indépendants (JL et LT) ont appliqué à ces articles les critères d'inclusion mentionnés plus haut. Le taux de concordance entre les paires de codeurs a été de 97,9 \% pour les titres et les résumés. Ont été exclus les articles qui traitaient de sujets non pertinents pour cette revue : déclarations rétrospectives par des adultes de maltraitance envers les enfants, 
FIGURE 1

Recension de l'information à travers les phases de la revue

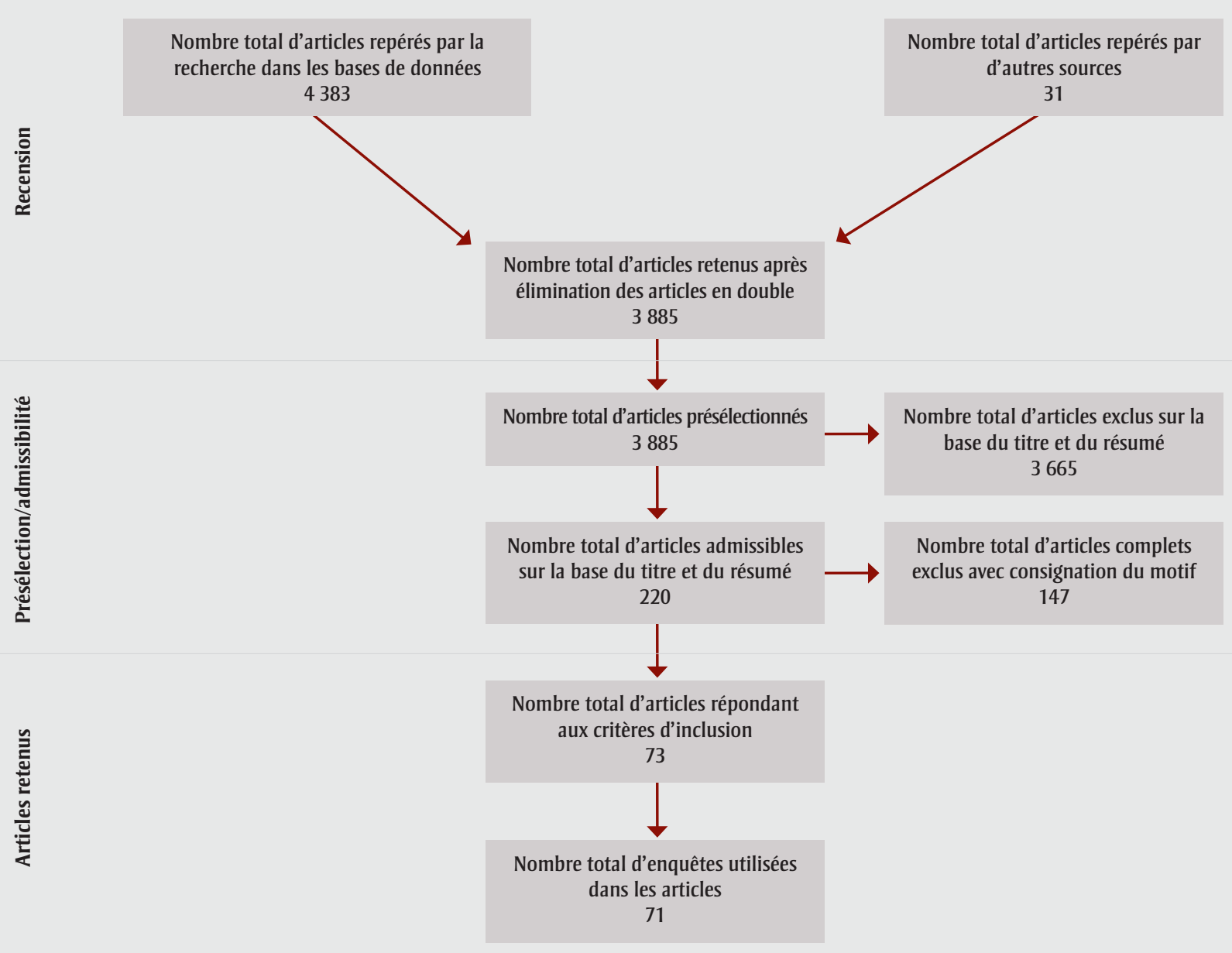

toxicomanie, échantillons non représentatifs, articles de journaux, résumés de congrès, commentaires et lettres à la rédaction. Chaque examinateur a également répertorié par type les cas de maltraitance signalés. Même si les définitions précises de la maltraitance envers les enfants variaient d'un article à l'autre, elles étaient suffisamment similaires sur le plan conceptuel pour qu'on puisse appliquer les classifications de l'Agence de la santé publique du Canada (ASPC) que sont la violence psychologique (VP), la négligence (NG), l'exposition à la violence conjugale (EVC) et l'abus physique et sexuel (AP et AS) ${ }^{21}$ (tableau 1).

Nous avons modifié une clé de codage utilisée pour évaluer l'exposition rétrospective d'adultes à la maltraitance envers les enfants $^{6}$. Si elles étaient mentionnées, la fiabilité et la validité des mesures de la maltraitance étaient notées. La documentation des procédures en matière d'éthique a porté sur les mesures prises pour préserver la confidentialité, pour offrir du soutien aux répondants ou pour atténuer leur détresse pendant ou après l'enquête (voir tableau 2). Nous avons également extrait des articles les renseignements relatifs à l'administration de l'enquête et aux mesures d'évaluation de la qualité des données. Nous avons consulté les sources externes (articles ou sites Internet) citées dans les articles pour obtenir l'information sur la validité et la fiabilité des mesures de la maltraitance envers les enfants et, dans certains cas, ces sources nous ont aussi permis de comprendre comment la maltraitance était définie pour la réalisation d'une enquête ou de préciser les procédures d'enquête. Dans le cas où les données provenant de la source externe ne concordaient pas avec celles de l'article retenu dans la revue, les dernières avaient préséance. Dans le cas où les données d'articles sélectionnés pour la revue et concernant la même enquête étaient contradictoires, c'est l'article se rapprochant le plus des objectifs de l'étude qui a été retenu.

Enfin, pour vérifier que les articles choisis répondaient aux critères d'inclusion et pour s'assurer de l'exactitude de toutes les données extraites, les articles ont été évalués par deux autres examinateurs (CW, SA ou JD) et tous les désaccords ont fait l'objet de discussions jusqu'à l'obtention d'un consensus.

\section{Résultats}

Nous avons sélectionné, à partir de leur titre et de leur résumé, 220 articles sur les 3885 repérés au cours de notre recherche en ligne. Sur ce nombre, 73 ont répondu aux critères d'inclusion et ils rendaient compte de 71 enquêtes. Le tableau 3 présente les caractéristiques de l'échantillon, 
TABLEAU 1

Définition de la maltraitance envers les enfants

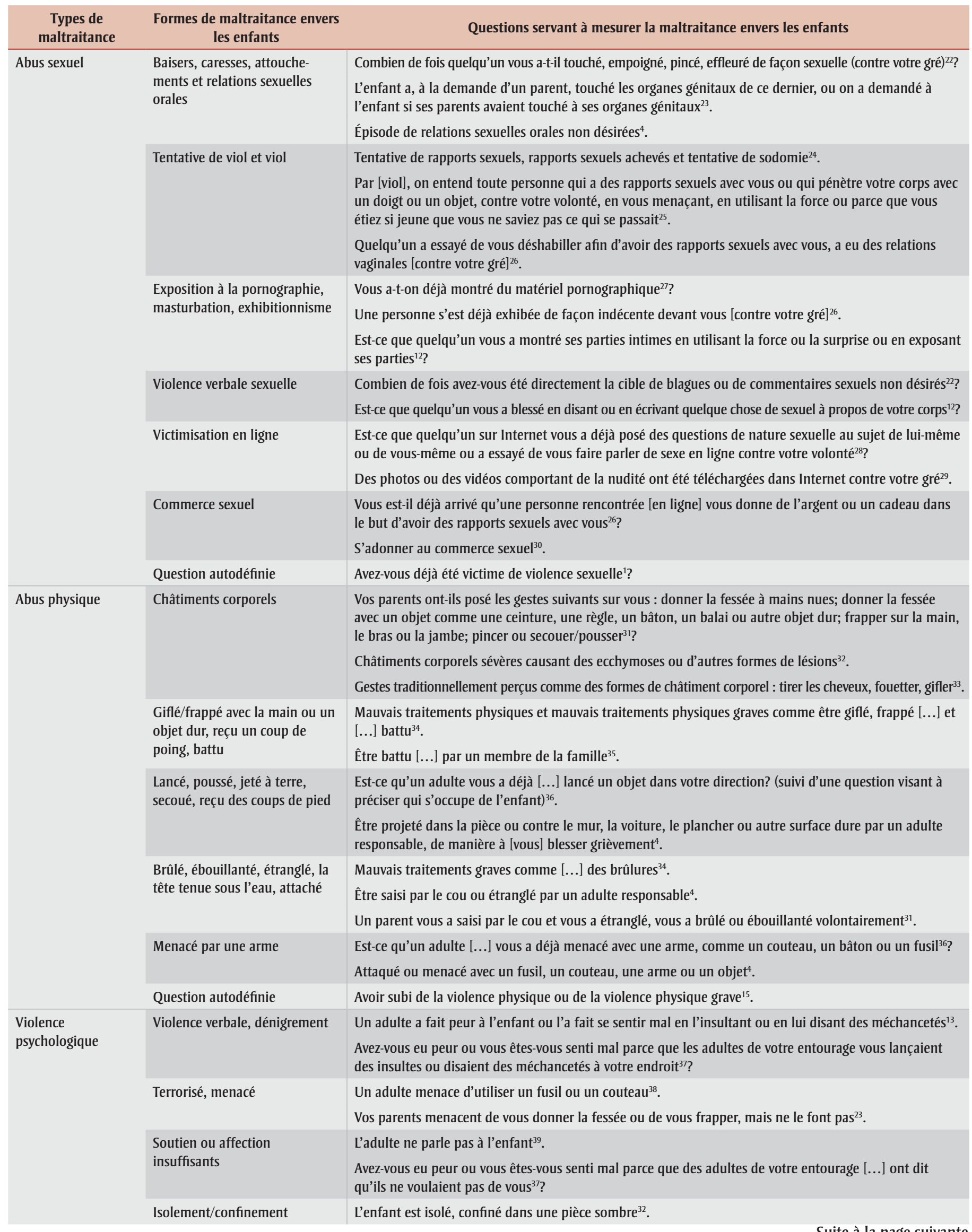


TABLEAU 1 (suite)

Définition de la maltraitance envers les enfants

\begin{tabular}{|c|c|c|}
\hline $\begin{array}{l}\text { Types de } \\
\text { maltraitance }\end{array}$ & $\begin{array}{l}\text { Formes de maltraitance envers } \\
\text { les enfants }\end{array}$ & Questions servant à mesurer la maltraitance envers les enfants \\
\hline \multirow[t]{3}{*}{ Négligence } & Absence de supervision & $\begin{array}{l}\text { Faire l'objet d'une supervision inadéquate et devoir effectuer des tâches inappropriées en raison de } \\
\text { l'âge }{ }^{40} \text {. }\end{array}$ \\
\hline & Physique & 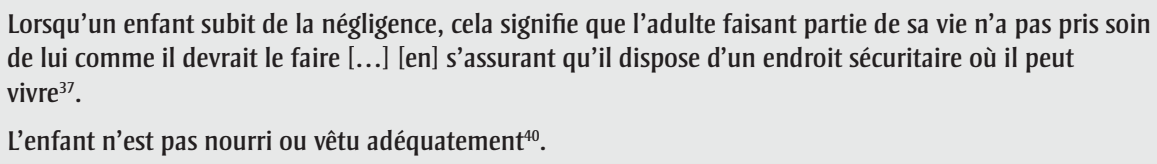 \\
\hline & Médicale & $\begin{array}{l}\text { Lorsqu'un enfant subit de la négligence, cela signifie que l'adulte faisant partie de sa vie n'a pas pris soin } \\
\text { de lui comme il devrait le faire [...] [en] l'amenant consulter un médecin lorsqu'il est malade }{ }^{37} \text {. }\end{array}$ \\
\hline \multirow[t]{2}{*}{$\begin{array}{l}\text { Exposition à la } \\
\text { violence conjugale }\end{array}$} & Violence physique & $\begin{array}{l}\text { La jeune personne a été témoin de violence physique entre ses parents }{ }^{41} \text {. } \\
\text { L'adolescent a vu ses parents se frapper à coups de poing, se frapper ou se battre, s'étrangler l'un l'autre, } \\
\text { se frapper mutuellement avec un objet. }\end{array}$ \\
\hline & Violence psychologique & $\begin{array}{l}\text { On a demandé à l'enfant s'il avait déjà [...] été témoin de disputes graves entre ses parents². } \\
\text { L'adolescent a vu ses parents [...] se menacer l'un l'autre avec un fusil, un couteau ou toute autre arme }{ }^{4} \text {. }\end{array}$ \\
\hline
\end{tabular}

la méthodologie de l'enquête, les mesures de la maltraitance envers les enfants, la fiabilité et la validité, le taux de réponse et les mesures prises pour améliorer le taux de réponse, les approches et les protocoles conçus pour réconforter les participants ou atténuer leur détresse et enfin les types de maltraitance envers les enfants. La plupart des données ont été recueillies dans les écoles. La collecte de données a été effectuée par l'entremise d'un questionnaire auto-administré le plus fréquemment, dans certains cas par une entrevue en personne, et moins fréquemment, par une entrevue par téléphone. Onze mesures ont été appliquées, souvent avec modification par rapport à la version d'origine. C'est le Juvenile Victimisation Questionnaire (JVQ - questionnaire de victimisation juvénile) qui a été utilisé le plus souvent (8 occurrences), suivi de différentes versions de l'Échelle des tactiques de résolution des conflits (CTS) (6 occurrences) et de l'Outil de dépistage de la maltraitance des enfants (ICAST-CH) de la Société internationale pour la prévention des mauvais traitements et négligences envers les enfants (ISPCAN) (4 occurrences). Trente-sept articles ne fournissaient aucune information sur les mesures précises utilisées. En outre, peu d'articles ont fourni des données sur la fiabilité et la validité des mesures employées. Les taux de réponse des participants variaient entre $40,4 \%$ et $99,9 \%$. Dans la majorité des articles, il était question de mesures prises pour réconforter les répondants, même si les renseignements précis sur les procédures visant à atténuer la détresse étaient rares.

TABLEAU 2

\section{Approches visant à réconforter le répondant et à accroître le taux de réponse}

\begin{tabular}{|c|c|}
\hline & Définitions \\
\hline \multirow{10}{*}{ 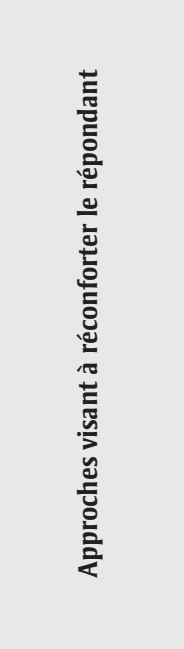 } & $\begin{array}{l}\text { Assentiment : Participant qui, d'un point de vue juridique, est trop jeune pour donner un consentement éclairé et qui manifeste sa volonté } \\
\text { de participer à la recherche, puisqu'il est assez vieux pour comprendre l'objet de la recherche. }\end{array}$ \\
\hline & Consentement : Accord volontaire d’une personne, ou de son représentant autorisé, qui a la capacité juridique de donner son consentement. \\
\hline & $\begin{array}{l}\text { Consentement actif : Le parent ou le tuteur légal est tenu de signer et de retourner un formulaire s'il consent à ce que son enfant participe à } \\
\text { la recherche. }\end{array}$ \\
\hline & $\begin{array}{l}\text { Consentement passif : Le parent ou le tuteur légal est tenu d'aviser l'école ou les chercheurs s'il refuse que son enfant participe à la } \\
\text { recherche. }\end{array}$ \\
\hline & Confidentialité : Mesures prises pour assurer la confidentialité des données après leur collecte. \\
\hline & $\begin{array}{l}\text { Protection des renseignements personnels : Mesures prises pour assurer la protection des renseignements personnels des répondants durant } \\
\text { la collecte de données. }\end{array}$ \\
\hline & Anonymat : Aucun renseignement permettant d’identifier les répondants n’a été recueilli. \\
\hline & $\begin{array}{l}\text { Milieu sécuritaire : Présence de figures rassurantes, comme les enseignants et les infirmiers, ainsi que d'éléments environnementaux pour } \\
\text { maximiser le confort des participants. }\end{array}$ \\
\hline & Participation volontaire : Le choix de participer à l'étude revenait au participant. \\
\hline & Retrait : Les participants ont été informés qu'ils pouvaient mettre fin à l'enquête en tout temps durant la collecte de données. \\
\hline \multirow{3}{*}{ 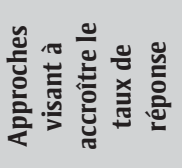 } & Incitatif : Récompense matérielle offerte en échange d’une participation à l’étude. \\
\hline & Temps pour remplir le questionnaire : Le temps nécessaire pour terminer l’enquête a été enregistré. \\
\hline & $\begin{array}{l}\text { Rappels : Les participants qui n'étaient pas disponibles au moment de la collecte de données ont été joints plus tard et ont eu la possibilité } \\
\text { de participer. }\end{array}$ \\
\hline
\end{tabular}




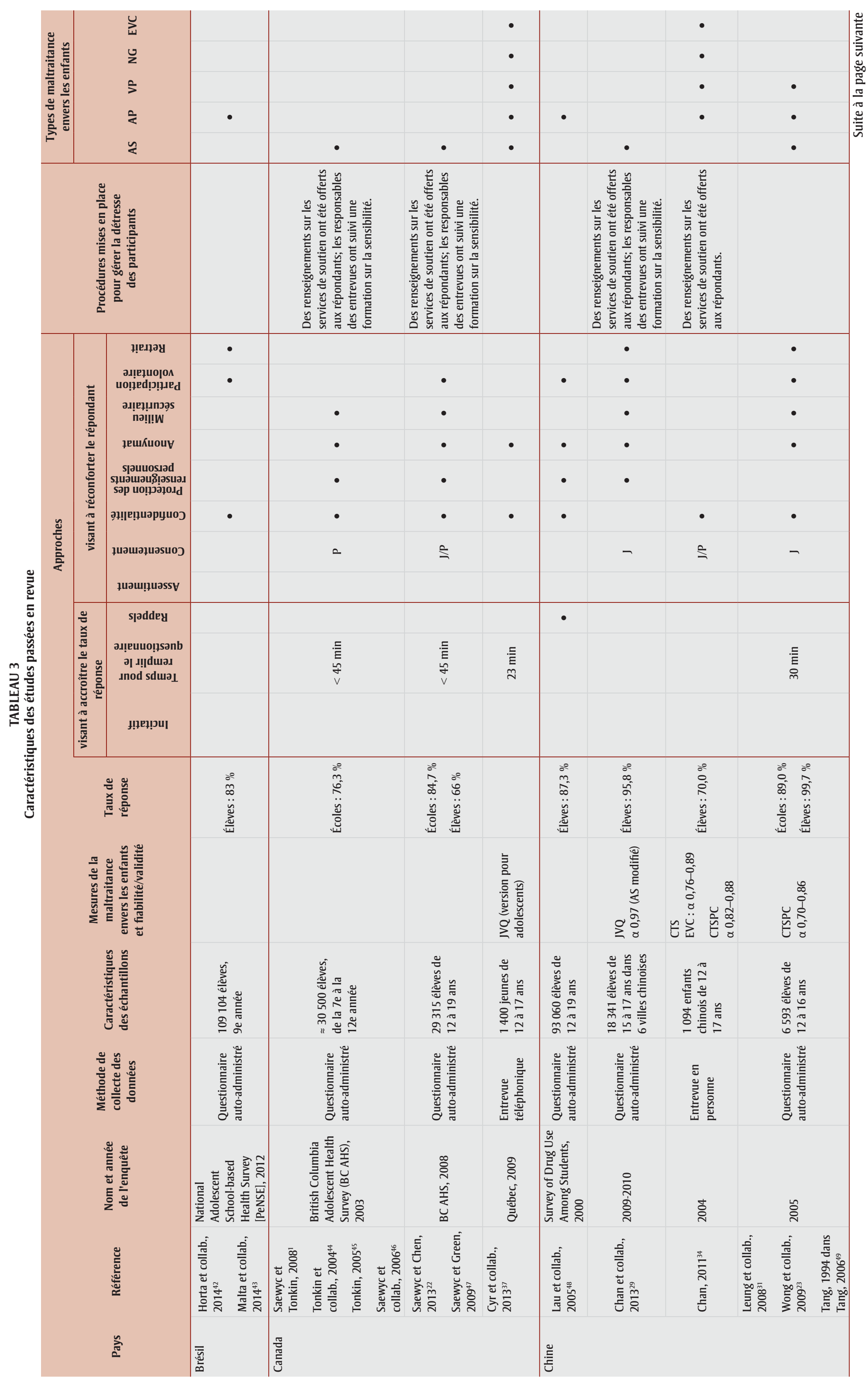




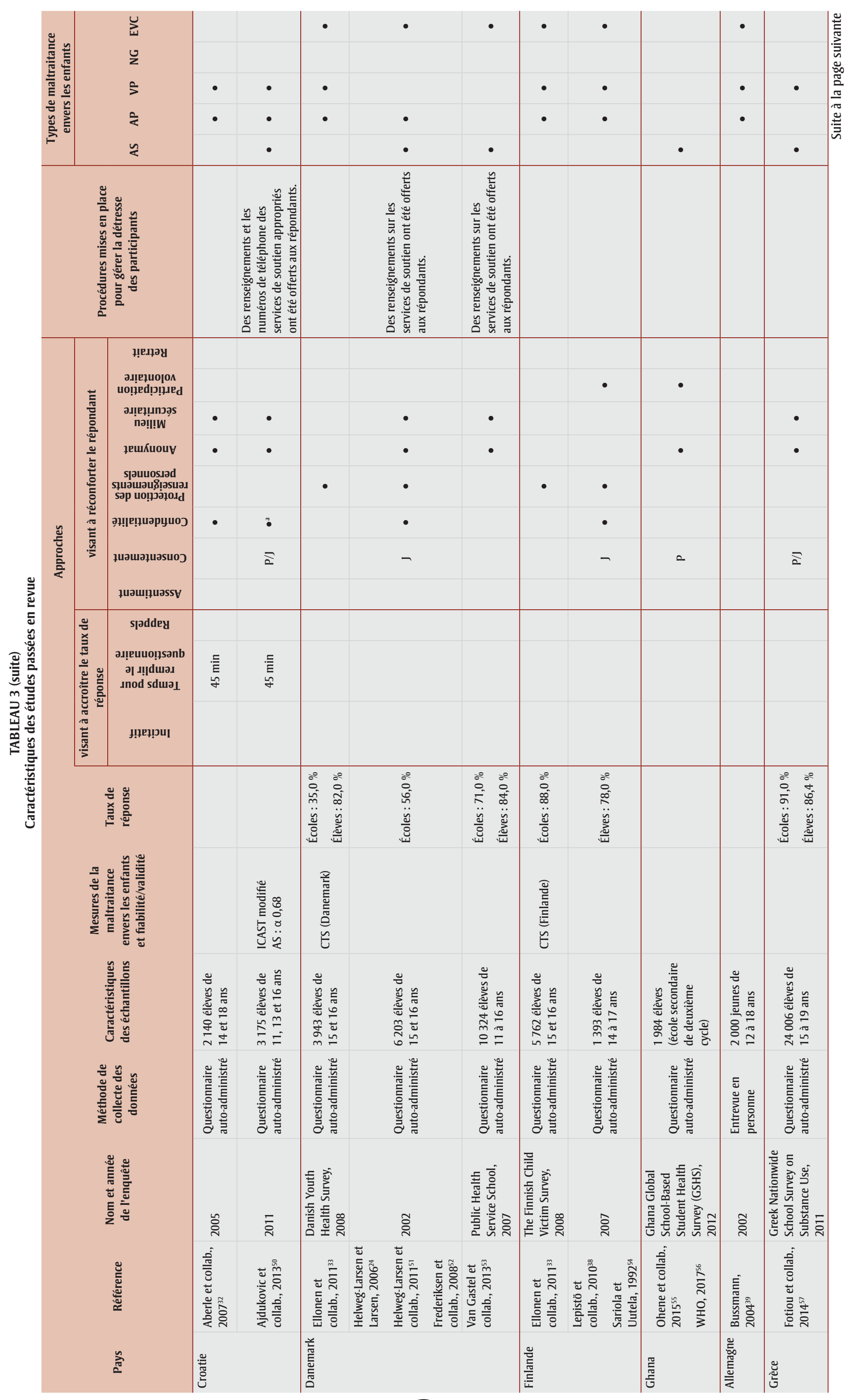




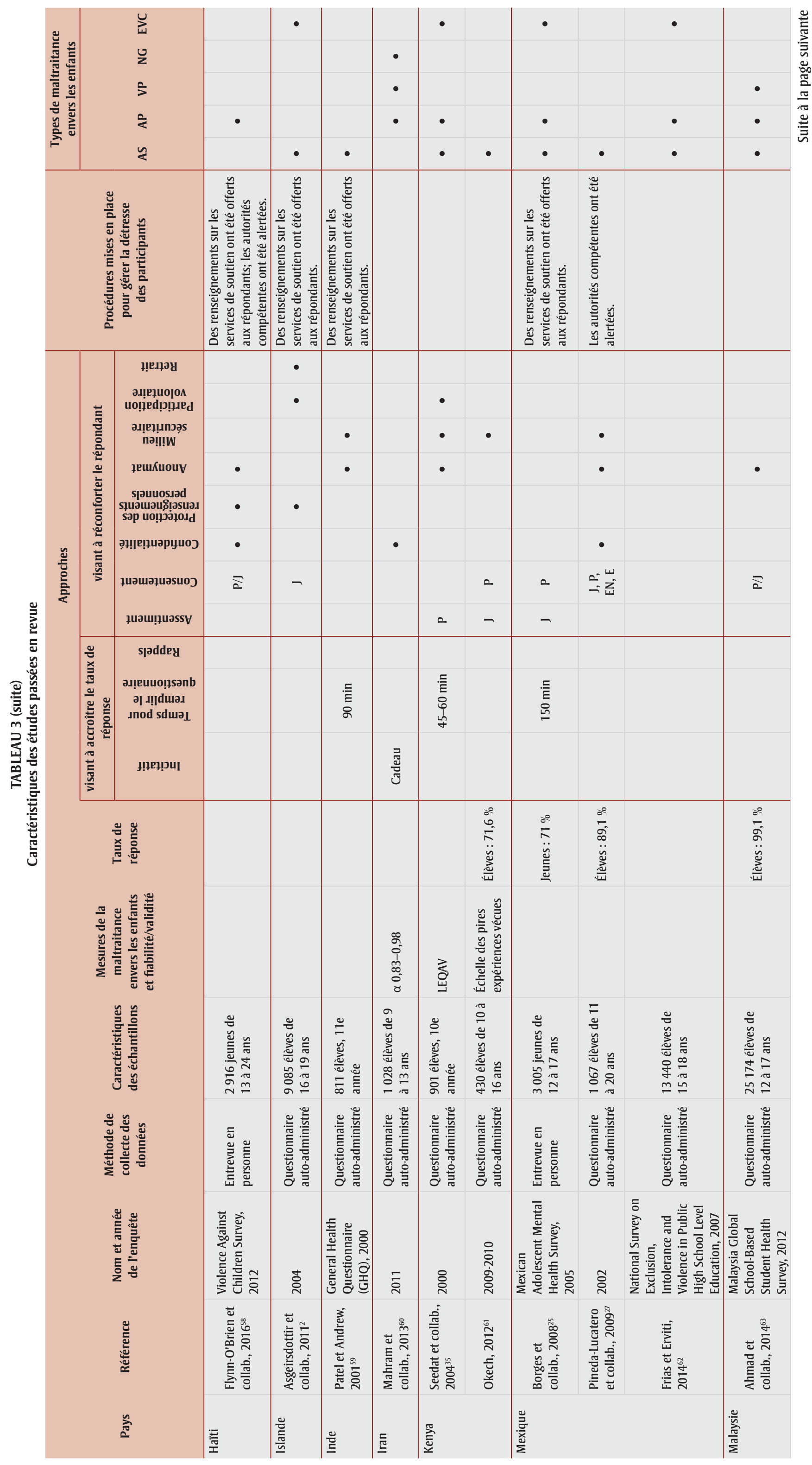




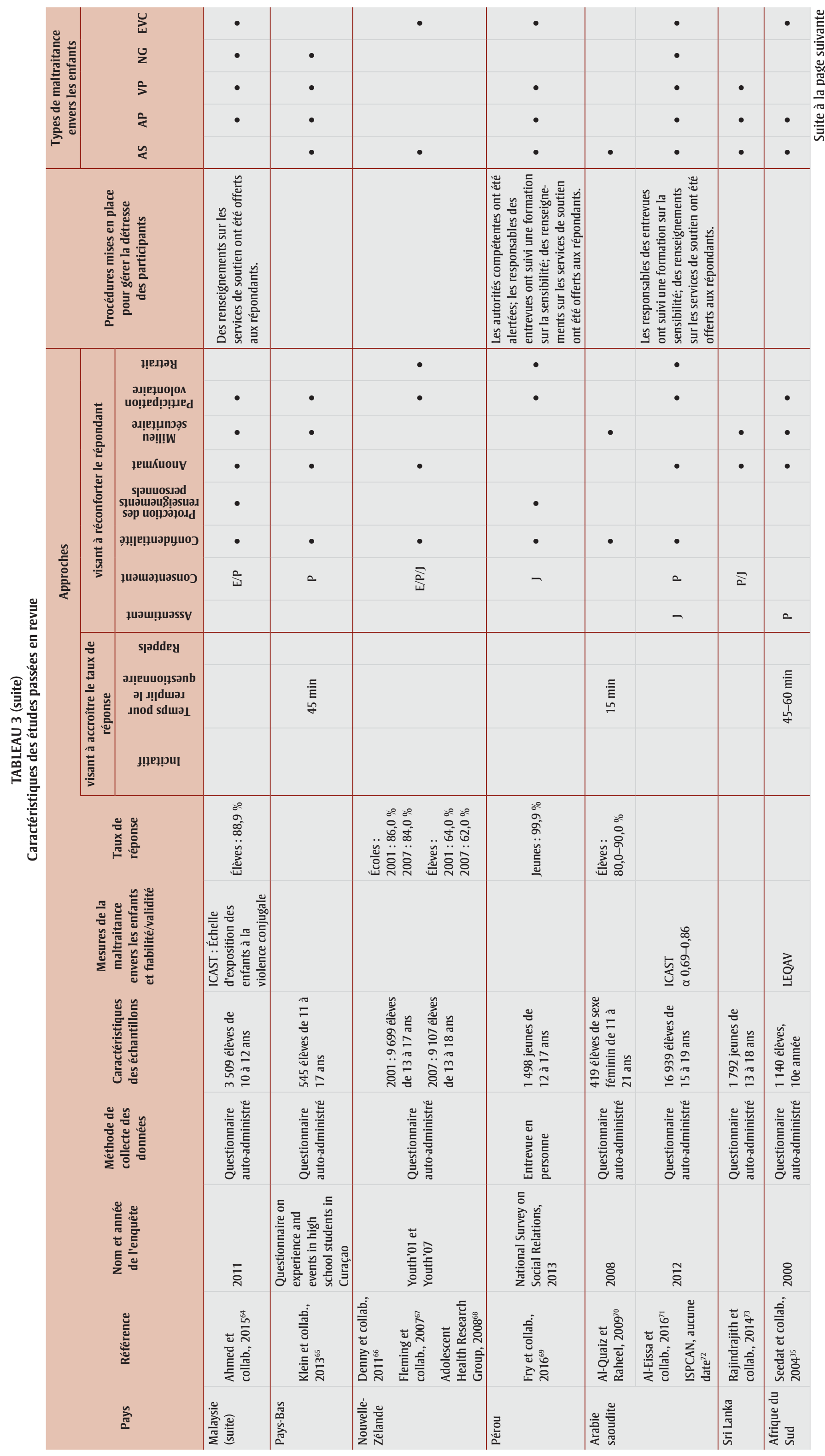




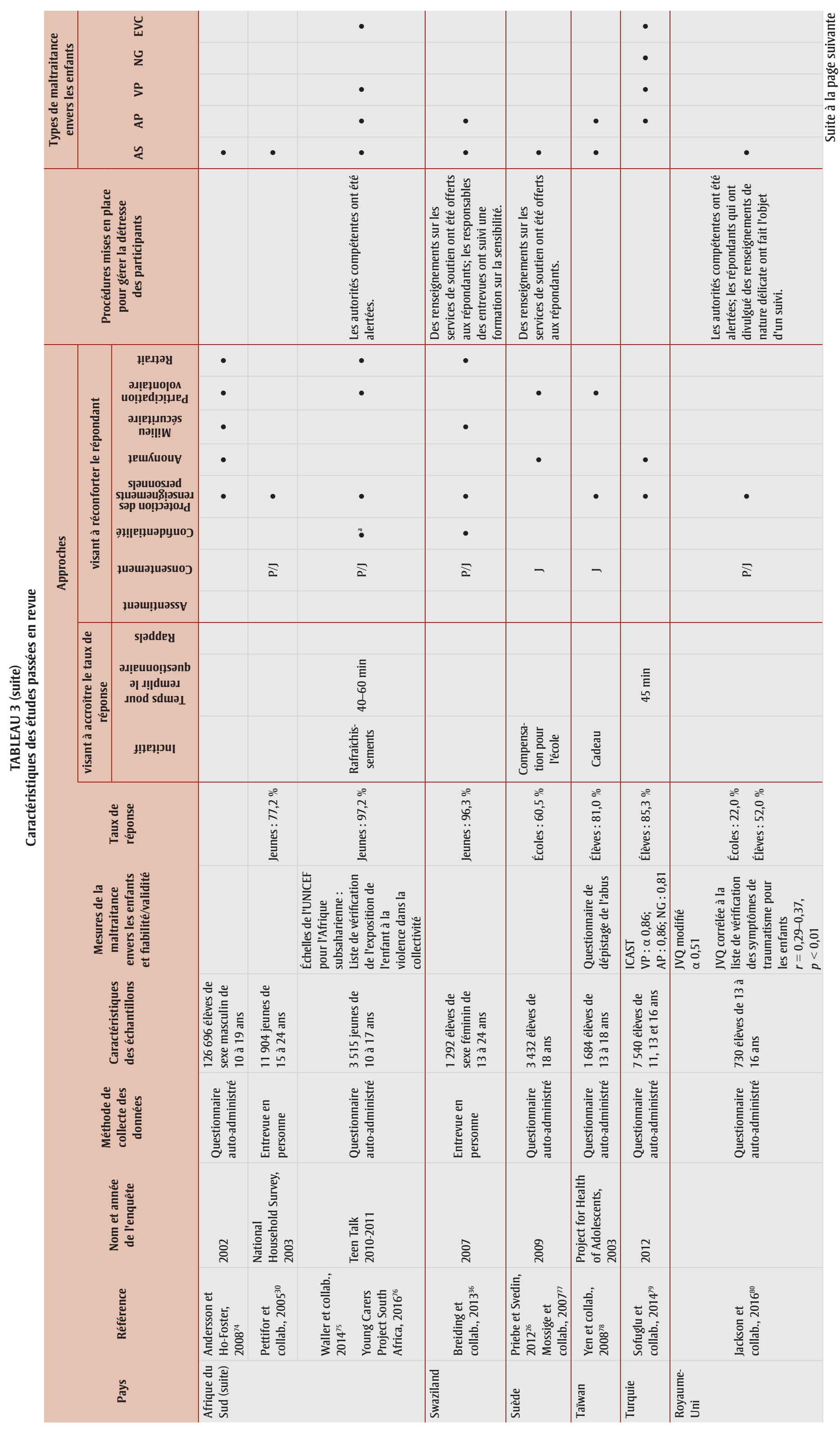




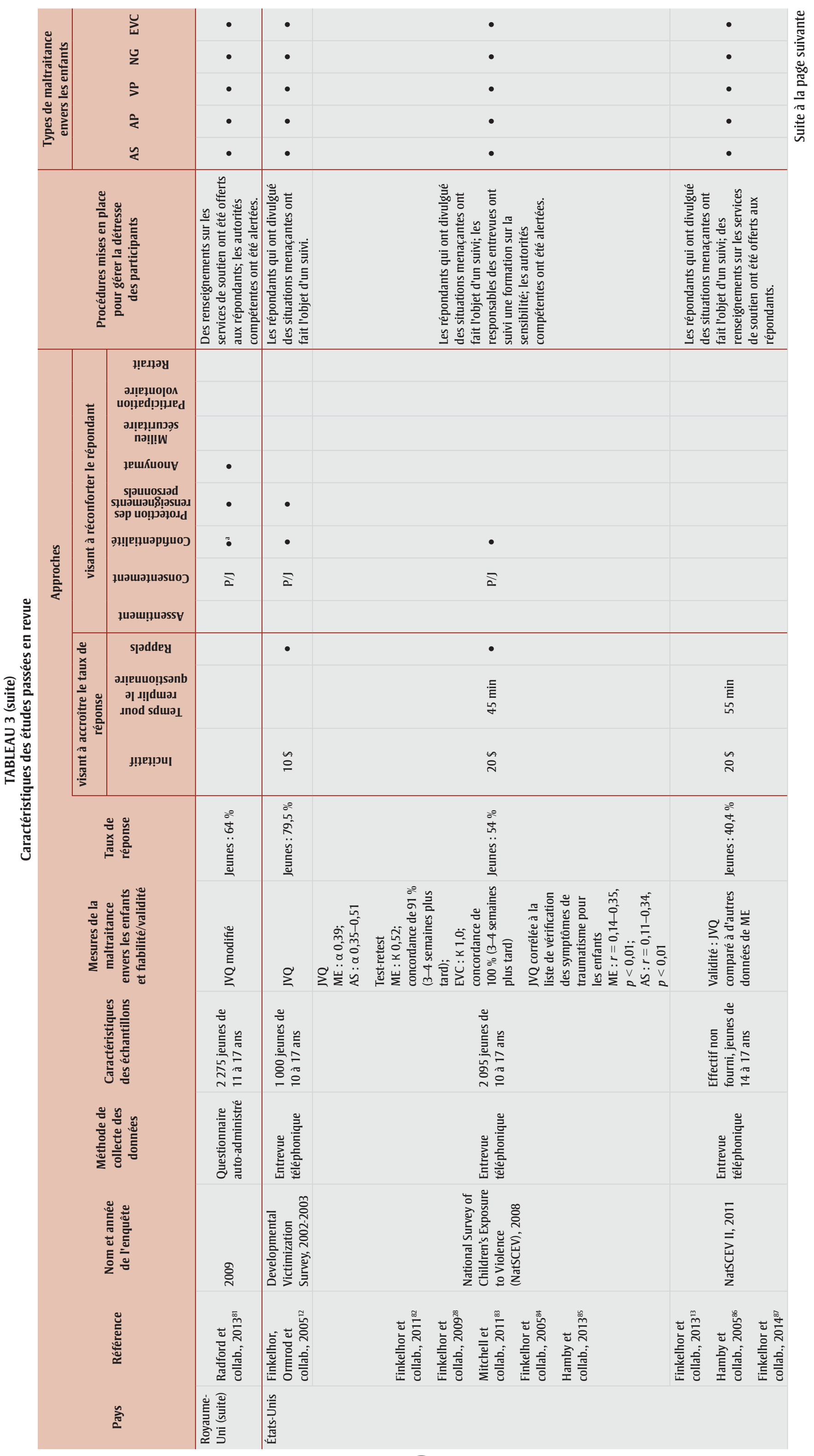




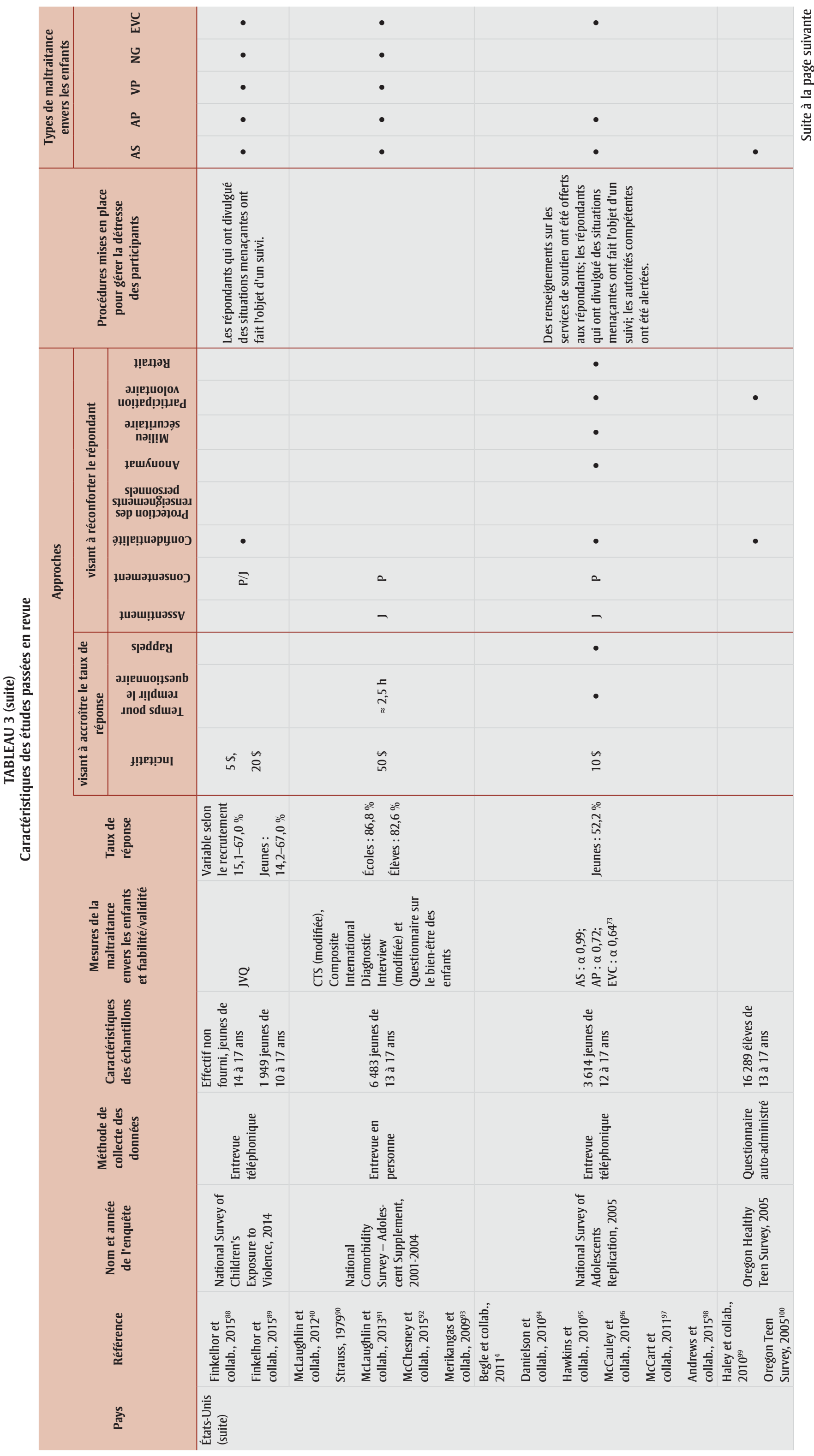




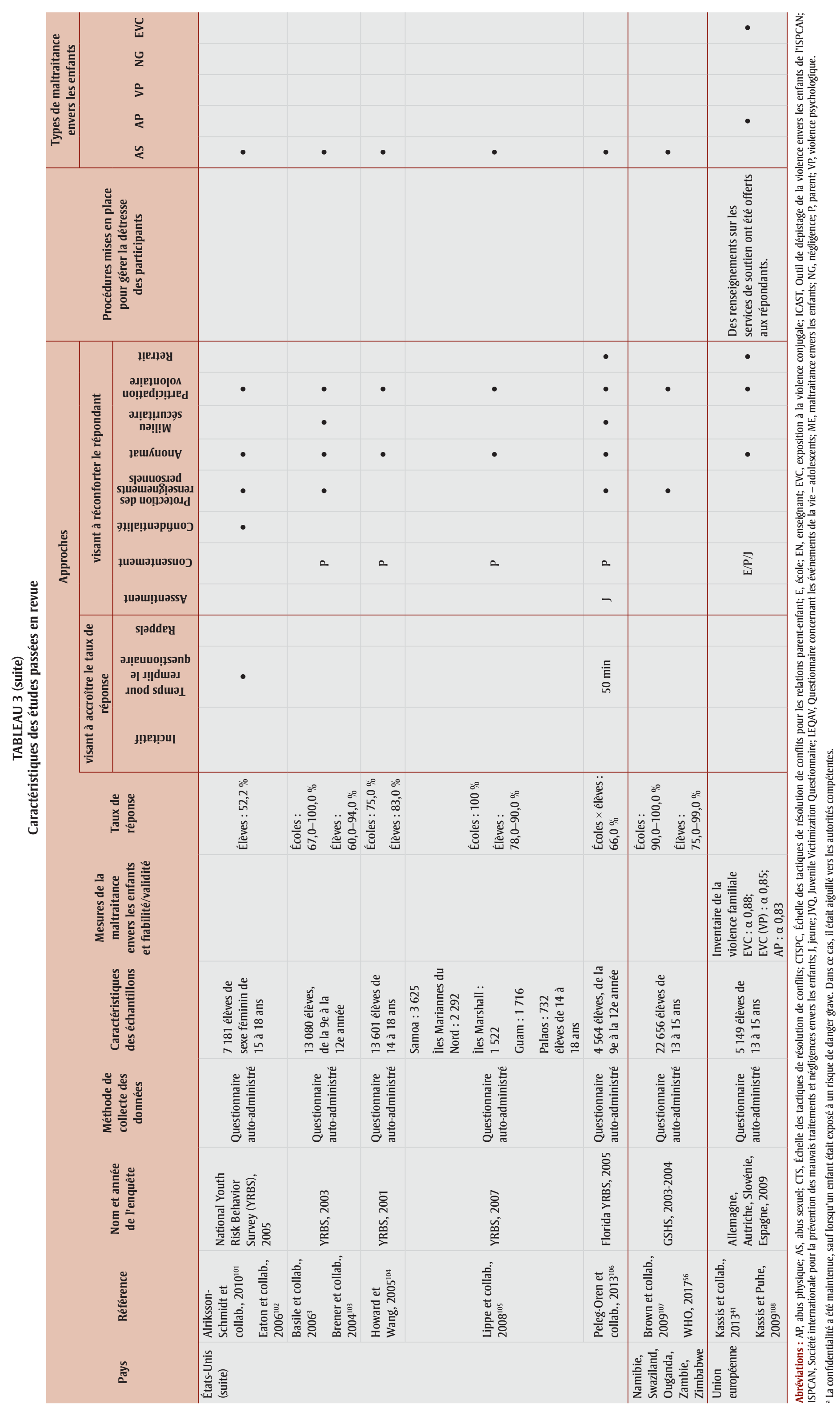


Les procédures les plus souvent mentionnées pour atténuer la détresse des participants ou y réagir étaient : 1) fournir aux répondants des informations et des numéros de téléphone en lien avec des services de soutien appropriés, 2) effectuer un suivi auprès des répondants ayant fait part de situations menaçantes, 3 ) offrir aux responsables des entrevues une formation ciblée sur la sensibilité et 4) alerter les autorités compétentes lorsqu'une intervention était jugée nécessaire. Bien sûr, informer les participants de la possibilité d'alerter les autorités était susceptible de nuire à la participation.

En matière de types de maltraitance, c'est l'abus sexuel qui a été le plus souvent mentionné dans les questions de l'enquête (tableau 3). Dans la majorité des mesures de la maltraitance, on avait prédéfini des comportements plutôt que laissé cours à l'autodéfinition, et l'abus sexuel avait été décrit de façon plus détaillée que les autres types. Les estimations de prévalence de la maltraitance envers les enfants variaient selon la mesure utilisée et n'étaient pas toujours mentionnées. L'hétérogénéité des mesures et la variation entre les périodes couvertes ont empêché toute comparaison valable entre les estimations de prévalence. Les estimations globales de prévalence au cours de la vie variaient entre $0,3 \%$ et $44,3 \%$ pour l'abus sexuel, entre $42 \%$ et $58,3 \%$ pour l'abus physique, entre 3,1\% et $78,3 \%$ pour la violence psychologique, entre $0,9 \%$ et $38,3 \%$ pour la négligence et entre $0,6 \%$ et $30,9 \%$ pour l'exposition à la violence conjugale.

\section{Analyse}

Les résultats de cette revue systématique mettent en lumière les efforts soutenus déployés pour mesurer la maltraitance envers les enfants à l'échelle de la population et, par là-même, l'importance accordée à ce problème à l'échelle de la planète. Cette revue nous a permis de cerner les diverses stratégies employées pour améliorer l'exactitude des données et atténuer la détresse des participants. Nos résultats sont similaires à ceux de la revue de $2000^{17}$. Tant nos résultats que ceux de 2000 démontrent qu'il est possible de recueillir des données sur la maltraitance envers les enfants en dépit de la persistance de problèmes d'uniformité des définitions.

\section{Recension des enquêtes et des facteurs ayant une influence sur les estimations de la prévalence}

Les estimations de la prévalence de la maltraitance des enfants varient considérablement entre les études examinées. Pour évaluer les résultats des différentes enquêtes, il est important de tenir compte des facteurs intrinsèques à l'autodéclaration susceptibles de compromettre la comparabilité24. Parmi ces obstacles figurent la culpabilisation, le développement cognitif et l'âge, la stigmatisation, la peur de subir des représailles de la part de l'auteur de la maltraitance et enfin l'incapacité à reconnaître le caractère abusif du comportement ${ }^{16}$. En ce qui concerne ce dernier élément, des perceptions différentes de ce qui constitue la limite entre l'exercice de la discipline et un mauvais traitement peuvent inciter le répondant à formuler des réponses incohérentes ${ }^{8}$. Dans certaines cultures, le recours aux châtiments corporels est courant voire accepté d'un point de vue juridique $^{31,39}$, tandis que dans d'autres cultures il s'agit d'un abus ${ }^{109}$. Dans certaines études, les comportements liés à l'abus sexuel n’ont pas été évalués car ce sujet a été jugé trop sensible sur le plan culturel $^{50,60}$.

Les écarts entre études dans les estimations de la prévalence de la maltraitance envers les enfants sont également attribuables à des différences dans les mesures. Par exemple, dans l'optique d'encourager la divulgation de l'abus sexuel, certaines enquêtes font appel à des questions précises en matière de comportement ${ }^{3}$, tandis que d'autres ont recours à des questions formulées en des termes plus généraux ${ }^{101}$. Certaines mesures de la maltraitance sont dichotomiques (oui-non), alors que d'autres exigent des précisions sur la gravité et la fréquence de l'abus.

Les différences en matière de conception peuvent aussi avoir une incidence sur les estimations de la prévalence. Par exemple, certaines enquêtes incluent explicitement la victimisation en ligne en tant que composante de l'abus sexuel alors que d'autres ne le font pas. Enfin, les variations dans le vocabulaire employé pour décrire certains comportements peut également avoir une influence sur la comparabilité. À titre d'exemple, l'expression " rapports sexuels forcés sans consentement " pourrait être interprétée dans un sens plus large que « viol » et serait donc plus susceptible de se traduire par une réponse positive (et accroître la prévalence apparente). Par ailleurs, la négligence a été mesurée dans quelques enquêtes seulement, ce qui témoigne peut-être des difficultés inhérentes à obtenir de l'information sur ce sujet dans les enquêtes fondées sur la population. Dans certaines communautés, les estimations relativement plus faibles de cas de négligence étaient attribuables à des réseaux sociaux et à des conditions de vie où règne la proximité ${ }^{65}$. Des efforts sont en cours pour améliorer la collecte de données sur la négligence dans les enquêtes de population effectuées auprès de jeunes répondants ${ }^{110,111}$.

\section{Qualité des données}

La majorité des articles examinés contenait peu de détails sur la fiabilité ou la validité des mesures utilisées dans les enquêtes. Des énoncés tels que " la fiabilité de l'échelle est bien documentée " ou indiquant que la validité a été vérifiée par les auteurs sont courants mais ne fournissent pas d'information vraiment pertinente. La validité n'a malheureusement été mentionnée que dans trois articles ${ }^{80,84,87}$. En matière de fiabilité, c'est la cohérence interne mesurée par l'alpha de Cronbach qui a été le plus souvent documentée, suivie de la fiabilité inter-évaluateurs exprimée en pourcentage de concordance.

La cohérence interne est peut-être d'une utilité limitée, étant donné qu'aucun lien n'existe entre certains comportements de maltraitance. Par exemple, certaines formes de négligence peuvent ne pas être liées entre elles ni liées à d'autres types de maltraitance envers les enfants. En raison des complexités relatives à la cohérence interne, cette mesure doit donc être interprétée avec prudence ${ }^{84,112}$. De manière générale, les enquêtes effectuées auprès des jeunes fournissent des données très peu touchées par le biais de rappel ${ }^{113}$. Bien entendu, la validité peut toujours être compromise par un biais de désirabilité sociale, du fait de la nature délicate des questions sur la maltraitance. Les recherches ont toutefois relevé peu de difficultés découlant de la nature délicate des questions posées $^{24,53,61}$. Les diffférences dans les stades de développement des mesures examinées pourraient partiellement expliquer pourquoi si peu de propriétés psychométriques des mesures de la maltraitance envers les enfants ont été mentionnées. Les mesures les plus récentes sont souvent 
ajustées pour tenir compte de la culture et de la langue, et la poursuite des essais devrait conduire à une amélioration de l'exactitude des données.

Les aspects techniques de la collecte de données et le contexte dans lequel celle-ci est réalisée ont également une incidence sur la qualité des données et le taux de réponse. La plupart des études passées en revue reposant sur des enquêtes menées dans les écoles (où tous les élèves répondent au même sondage en même temps), les taux de réponse obtenus sont élevés. En revanche, dans les écoles, la volonté de participer n'a pas été partout la même, pour des raisons indépendantes des questions sur la maltraitance envers les enfants $^{33,53,57}$. Les travaux de recherche semblent indiquer que, pour les élèves, le fait de protéger au maximum les renseignements personnels et de garantir l'anonymat est efficace pour obtenir des taux de réponse élevés ${ }^{45}$. L'importance que revêt le respect de la vie privée a aussi été soulignée dans une étude dans laquelle on a constaté que des participants plus jeunes (âgés de 10 ans) étaient davantage troublés lorsqu'ils répondaient en présence d'un accompagnateur que lorsqu'ils étaient seuls $^{114}$.

Les moyens employés pour obtenir le consentement à participer à une enquête peuvent également avoir une incidence sur le taux de réponse : la nécessité d'obtenir le consentement des parents peut dissuader les jeunes de participer, surtout ceux ayant subi de la maltraitance ${ }^{47,51,115}$. Le consentement passif des parents a été utilisé dans de nombreuses enquêtes pour accroître le taux de réponse et éviter un biais d'échantillonnage lié au consentement parental actif ${ }^{65,80,106}$. Dans le cadre d'une étude, des chercheurs ont conçu et utilisé une procédure de consentement modifiée en cas de mauvais traitements subis par les répondants de la part de leur pourvoyeur de soins principal ${ }^{58}$.

\section{Considérations d'ordre éthique}

L'obtention de renseignements sur les expériences associées à la maltraitance envers les enfants est une tâche délicate : la manière dont les questions sont formulées est importante. Même le titre de l'enquête peut créer de l'anxiété et entraîner un refus de participation (p. ex. une enquête sur " la maltraitance envers les enfants » peut provoquer des émotions plus fortes qu'une enquête sur " la santé des enfants »). De même, les termes employés dans les questions sur l'expérience de maltraitance envers les enfants peuvent influencer le répondant. Être sensible aux réactions potentielles de rejet est essentiel, tout comme l'est un énoncé clair assurant l'anonymat et la confidentialité de l'enquête. Notre revue a toutefois permis de constater que certains chercheurs avaient inclus dans le formulaire de consentement une procédure de non-respect de la confidentialité au cas où un jeune aurait besoin d'une protection, procédure permettant de l'aiguiller automatiquement vers les autorités compétentes ${ }^{50,75,81}$, et cette stratégie n'a pas nui au taux de réponse $\mathrm{e}^{75,81}$.

Notre revue systématique laisse penser que les jeunes sont généralement à l'aise pour répondre aux questions sur leur expérience de la maltraitance $e^{12,14,71,116}$. Selon une étude, 4,6 \% des jeunes ont déclaré avoir été contrariés lorsqu'ils ont répondu à une enquête sur la maltraitance envers les enfants, mais 95,3\% de ces derniers ont dit qu'ils participeraient néanmoins à une enquête similaire $^{116}$. Fait intéressant, sur les 17,3 \% de participants ayant déclaré avoir vécu des expériences considérées comme à risque élevé, seulement $2 \%$ ont été aiguillés vers des services de counseling ${ }^{116}$. En outre, un article a mentionné que $11 \%$ des participants n'avaient pas répondu aux questions sur l'abus sexuel, mais sans fournir d'information permettant d'évaluer si le nombre de refus de réponse était plus élevé pour les questions portant sur l'abus sexuel que pour les autres ${ }^{2}$. Plusieurs chercheurs sont parvenus à la conclusion que les avantages potentiels à obtenir de l'information sur la maltraitance envers les enfants dépassaient le risque de détresse éprouvée par le répondant ${ }^{7,116,117}$. Par exemple, une étude réalisée auprès d'adolescents comparant le stress produit par des questions sur la maltraitance à celui découlant de questions sur les notes à l'école n'a révélé aucune différence ${ }^{118}$.

\section{Limites}

Notre revue présente plusieurs limites. Premièrement, des incohérences dans les mesures de la maltraitance envers les enfants entre les enquêtes, et parfois même entre les cycles d'une même enquête, ont rendu la classification difficile. Deuxièmement, certains articles qui par ailleurs répondaient aux critères d'inclusion ont été exclus sur la base d'une information insuffisante en matière de méthodologie. Par exemple, on a choisi de ne pas inclure plusieurs articles pour lesquels il a été impossible d'établir une relation entre l'auteur de la maltraitance et la victime ou de faire une distinction entre l'exposition à la violence familiale et l'exposition à la violence dans la collectivité. Troisièmement, les estimations de la prévalence ne sont pas présentées de manière normalisée. Quatrièmement, comme il n'a souvent pas été possible de distinguer les mesures prises pour augmenter le taux de réponse de celles prises pour améliorer le confort des répondants, ces deux types de mesure ont été examinés conjointement. Cinquièmement, les mesures ont souvent été modifiées par rapport à leur version d'origine, en général sans que les résultats des essais de validité et de fiabilité des versions modifiées n'aient été fournis. Sixièmement, certains segments de la population ont été exclus, soit parce qu'ils ne fréquentaient pas l'école, soit parce qu'ils étaient absents le jour de la collecte de données. Septièmement, l'exclusion des articles rédigés dans d'autres langues que l'anglais a limité la portée internationale de cette revue. Huitièmement, seuls les articles évalués par les pairs ont fait partie de la revue, un procédé susceptible d'introduire un biais de publication. Enfin, le fait de restreindre l'analyse à des articles sans examiner les enquêtes sous-jacentes a probablement mené à l'exclusion de certains renseignements pertinents.

\section{Répercussions}

Notre revue montre que, même si nous manquons de données nationales au Canada, la maltraitance envers les enfants est une préoccupation commune à bon nombre de sociétés et de cultures. Comme en témoigne le grand nombre d'enquêtes et d'études d'auto-évaluation questionnant les jeunes sur leur niveau d'aise, on peut recueillir de façon responsable et éthique auprès d'eux des données sur la maltraitance des enfants tout en protégeant leur santé et leur bien-être ${ }^{14,116}$. Les travaux de recherche et de surveillance en matière de maltraitance envers les enfants bénéficieraient grandement de l'inclusion systématique de questions sur ce sujet au sein des enquêtes d'auto-évaluation portant sur la santé des populations. Hovdestad et Tonmyr ${ }^{119}$ insistent sur l'importance de préparer la voie à l'inclusion de questions sur la maltraitance envers les enfants dans les enquêtes a) en se préparant à faire face à une résistance précoce, b) en s'assurant 
d'avoir un important soutien d'une variété de parties intéressées, c) en prenant connaissance de la littérature sur le sujet (dont les problèmes abordés dans cet article) et d) en demeurant prêt à faire des compromis et à montrer sa détermination. Des données recueillies sur une base régulière nous fourniraient l'occasion de mieux comprendre le fardeau et les facteurs en lien avec la maltraitance envers les enfants ${ }^{120}$. Les écoles constitueraient un excellent endroit pour la collecte de données, compte tenu de la forte participation associées à ces enquêtes et du taux de recrutement élevé chez les jeunes. Au terme des discussions et des ententes requises avec les autorités scolaires pertinentes, il serait facile de mettre en place des procédures en vue d'obtenir le consentement des jeunes à participer et le consentement passif des parents ou des tuteurs. Pour maximiser la qualité des données, les mesures servant à leur collecte devraient être soumises à des essais de fiabilité et de validité, avec une mention de l'ensemble des aspects méthodologiques de l'enquête. Il est également recommandé de formuler des questions axées sur le comportement, avec un choix de réponses permettant de recueillir des données sur la gravité et la fréquence de la maltraitance.

Des protocoles tenant compte de la détresse potentielle des participants sont indispensables, et les responsables des entrevues doivent être formés à mener des recherches avec sensibilité et à-propos. Les moyens efficaces d'évaluation de la détresse des participants doivent être perfectionnés et mis en application, et les résultats de ces évaluations devraient ensuite orienter la conception et le libellé des questionnaires. Les enquêtes devraient suivre un code d'éthique strict, dont les objectifs généraux devraient être la protection des renseignements personnels et de la confidentialité ainsi que le respect des répondants.

\section{Remerciements}

Les auteurs tiennent à remercier Kathryn Wilkins, Tanya Pires, Tanya Lary et Jaskiran Kaur d'avoir participé à la préparation de cet article et formulé des commentaires sur les versions précédentes.

\section{Conflits d'intérêts}

Il n’y a aucun conflit d'intérêts à signaler.

\section{Contribution des auteures et avis}

LT a conçu cette étude. CW, LT et JL ont rédigé le document : LT a rédigé le protocole, avec l'apport des autres auteurs. JL, LT, CW, JD et SA ont extrait et catégorisé les données. LT a dirigé le volet d'évaluation.

Le contenu de cet article et les opinions qui y sont exprimées n'engagent que les auteurs et ne sont pas forcément représentatifs de la position du Gouvernement du Canada.

\section{Références}

1. Saewyc EM, Tonkin R. Surveying adolescents: focusing on positive development. Paediatr Child Health. 2008; 13(1):43-47.

2. Asgeirsdottir BB, Sigfusdottir ID, Gudjonsson GH, Sigurdsson JF. Associations between sexual abuse and family conflict/violence, self-injurious behavior, and substance use: the mediating role of depressed mood and anger. Child Abuse Negl. 2011;35(3): 210-219. doi: 10.1016/j.chiabu.2010.12 .003 .

3. Basile KC, Black MC, Simon TR, et al. The association between self-reported lifetime history of forced sexual intercourse and recent health-risk behaviors: findings from the 2003 national youth risk behavior survey. J Adolesc Health. 2006;39(5):752.e1-7.

4. Begle AM, Hanson RF, Danielson CK, McCart MR, Ruggiero KJ, Amstadter $\mathrm{AB}$, et al. Longitudinal pathways of victimization, substance use, and delinquency: findings from the national survey of adolescents. Addict Behav. 2011;36(7):682-689. doi: 10.1016 /j.addbeh.2010.12.026.

5. Rhodes AE, Boyle MH, Bethell J, Wekerle C, Goodman D, Tonmyr L, et al. Child maltreatment and onset of emergency department presentations for suicide-related behaviours. Child Abuse Negl. 2012;36(6):542-551.

6. Hovdestad W, Campeau A, Potter D, Tonmyr L. A systematic review of childhood maltreatment assessments in population-representative surveys since 1990. PLOS ONE. 2015;10(5). doi: 10.1371/journal.pone.0123366.
7. Tonmyr L, Hovdestad WE, Draca J. Commentary on Canadian child maltreatment data. J Interpers Violence. 2014;29(1):186-197.

8. Elliott K, Urquiza A. Ethnicity, culture, and child maltreatment. J Soc Issues. 2006;62(4):787-809.

9. Garbarino J, Ebata A. The significance of ethnic and cultural differences in child maltreatment. J Marriage Fam. 1983;45:773-83. doi: 10.2307/351790.

10. Smith C. Ethical considerations for the collection, analysis and publication of child maltreatment data. International Society for the Prevention of Child Abuse and Neglect. 2016.

11. Riley AW. Evidence that school-age children can self-report on their health. Ambul Pediatr. 2004;4(4): 371-6. doi: 10.1367/A03-178R.1.

12. Finkelhor D, Omrod R, Turner H, Hamby S. The victimization of children and youth: a comprehensive national survey. Child Maltreat. 2005; 10(1):5-25.

13. Finkelhor D, Turner HA, Shattuck A, Hamby SL. Violence, crime and abuse exposure in a national sample of children and youth: an update. JAMA Paediatr. 2013;167(7):614-621. doi: 10.1001/jamapediatrics.2013.42.

14. Helweg-Larsen K, Boving-Larsen $\mathrm{H}$. Ethical issues in youth surveys: potentials for conducting a national questionnaire study on adolescent schoolchildren's sexual experiences with adults. Am J Public Health. 2003; 93(11);1878-1882.

15. Helweg-Larsen K, Sundaram V, Curtis T, Boving Larsen $H$. The Danish Youth Survey 2002: Asking young people about sensitive issues. Int J Circumpol Heal. 2004;63(S2):147-152.

16. Colin-Vézina D, De La SablonniereGriffin M, Palmer AM, Milne L. A preliminary mapping of individual, relational and social factors that impede disclosure of childhood sexual abuse. Child Abuse Negl. 2015;43:12334. doi: 10.1016/j.chiabu.2015.03.010. 
17. Amaya-Jackson L, Socolar RRS, Hunter W, Runyan DK, Colindres, R. Directly questioning children and adolescent about maltreatment: a review of surveys measures used. J Interpers Violence. 2000;15(7):725-759.

18. Moher D, Liberati A, Tetzlaff J, Altman DG. The PRISMA Group (2009) Preferred reporting items for systematic reviews and meta-analyses: The PRISMA statement. PLOS Medicine. 2009;6(7):e1000097.

19. Fergusson DM, McLeod GF, Horwood LJ. Childhood sexual abuse and adult developmental outcomes: findings from a 30-year longitudinal study in New Zealand. Child Abuse Negl. 2013; 37(9):664-674.

20. MacMillan HL, Tanaka M, Duku E, Vaillancourt T, Boyle MH. Child physical and sexual abuse in a community sample of young adults: results from the Ontario Child Health Study. Child Abuse Negl. 2013;37(1):14-21. doi: 10.1016/j.chiabu.2012.06.005.

21. Agence de la santé publique du Canada. Étude canadienne sur l'incidence des signalements des cas de violence et de négligence envers les enfants : données principales. Ottawa (Ontario) : Agence de la santé publique du Canada; 2010.

22. Saewyc EM, Chen W. To what extent can adolescent suicide attempts be attributed to violence exposure? A population-based study from western Canada. Can J Commun Ment Health. 2013;32(1):79-94.

23. Wong WCW, Leung PWS, Tang CSK, et al. To unfold a hidden epidemic: prevalence of child maltreatment and its health implications among high school students in Guangzhou, China. Child Abuse Negl. 2009;33(7):441-450. doi: 10.1016/j.chiabu.2008.02.010.

24. Helweg-Larsen K, Larsen, HB. The prevalence of unwanted and unlawful sexual experiences reported by Danish adolescents: results from a national youth survey in 2002. Acta Paediatr. 2006;95:1270-6. doi: 10.1080/0803525 0600589033.
25. Borges G, Benjet C, Medina-Mora ME, Orozco R, Molnar BE, Nock MK. Traumatic events and suicide-related outcomes among Mexico City adolescents. J Child Psychol Psyc. 2008; 49(6):654-666. doi: 10.1111/j.1469 $-7610.2007 .01868 . x$.

26. Priebe G, Svedin CG. Online or off-line victimisation and psychological wellbeing: a comparison of sexual-minority and heterosexual youth. Eur Child Adoles Psyc. 2012;21(10):569-582. doi: 10.1007/s00787-012-0294-5.

27. Pineda-Lucatero AG, Trujillo-Hernández B, Millán-Guerrero RO, Vásquez C. Prevalence of childhood sexual abuse among Mexican adolescents. Child Care Health Dev. 2009;35(2):184-189.

28. Finkelhor D, Turner H, Ormrod R, Hamby SL. Violence, abuse, and crime exposure in a national sample of children and youth. Pediatrics. 2009; 124(5):1411-1423.

29. Chan KL, Yan E, Brownridge DA, Ip P. Associating child sexual abuse with child victimization in China. J Pediatr. 2013;162:1028-1034. doi: 10.1016/j. jpeds.2012.10.054.

30. Pettifor AE, Rees HV, Kleinschmidt I, Steffenson AE, MacPhail C, HlongwaMadikizela L, et al. Young people's sexual health in south Africa: HIV prevalence and sexual behaviors from nationally representative household survey. AIDS. 2005;19:1525-1534.

31. Leung PWS, Wong WCW, Chen WQ, Tang CSK. Prevalence and determinants of child maltreatment among high school students in southern China: a large scale school based survey. Child Adolesc Psychiatry Ment Health. 2008;2:27. doi: 10.1186/1753 -2000-2-27.

32. Aberle N, Ratković-Blažević V, Mitrović-Dittrich D, Coha R, Stoić A, Bublić J, et al. Emotional and physical abuse in family: survey among high school adolescents. Croat Med J. 2007;48(2):240-8.

33. Ellonen N, Kääriäinen $J$, Sariola $H$, Helweg-Larsen K, Larsen HB. Adolescents' experiences of parental violence in Danish and Finnish families: a comparative perspective. J Scand Stud Criminol Crime Prev. 2011;12(2):173197. doi: 10.1080/14043858.2011.622076.
34. Chan KL. Children exposed to child maltreatment and intimate partner violence: a study of co-occurrence among Hong Kong Chinese families. Child Abuse Negl. 2011;35:532-542. doi: 10.1016/j.chiabu.2011.03.006.

35. Seedat S, Nyamai C, Njenga F, Vythilingum, B, Stein DJ. Trauma exposure and post-traumatic stress symptoms in urban African schools: survey in Cape Town and Nairobi. Brit J Psychiat. 2004;184:169-175.

36. Breiding MJ, Mercy JA, Gulaid J, Reza A, Hleta-Nkambule N. A national survey of childhood physical abuse among females in Swaziland. J Epidemiol Glob Health. 2013;3(2):7381. doi: 10.1016/j.jegh.2013.02.006.

37. Cyr K, Chamberland C, Clement M, Lessard G, Wemmers J, Collin-Vézina $\mathrm{D}$, et al. Polyvictimization and victimization of children and youth: results from a population survey. Child Abuse Negl. 2013;37:814-820. doi: 10.1016/j .chiabu.2013.03.009.

38. Lepistö S, Åstedt-Kurki P, Joronen K, Luukkaala T, Paavilainen E. Adolescents' experiences of coping with domestic violence. J Adv Nurs. 2010; 66(6):1232-1245. doi: 10.1016/j.chiabu .2013.03.009.

39. Bussmann K. Evaluating the subtle impact of a ban on corporal punishment of children in Germany. Child Abuse Rev. 2004;13(5):292-311. doi: 10.1002/car.866.

40. McLaughlin KA, Green JG, Gruber MJ, Sampson NA, Zaslavsky AM, Kessler RC. Childhood adversities and first onset of psychiatric disorders in a national sample of US adolescents. Arch Gen Psychiatry. 2012;69(11):11511160. doi: 10.1001/archgenpsychiatry .2011.2277.

41. Kassis W, Artz S, Scambor C, Scambor E, Moldenhauer S. Finding the way out: a non-dichotomous understanding of violence and depression resilience of adolescents who are exposed to family violence. Child Abuse Negl. 2013;37(2):181-189. doi: 10.1016/j. chiabu.2012.11.001. 
42. Horta RL, Horta BL, Costa AWN, et al. Lifetime use of illicit drugs and associated factors among Brazilian schoolchildren, National Adolescent Schoolbased Health Survey (PeNSE 2012). Revista Brasileira de Epidemiologia. 2014;17:s31-45. doi: 10.1590/1809 -4503201400050004 .

43. Malta DC, Mascarenhas MDM, Dias AR, Prado RRd, Lima CM, Silva, MM.A da, et al. Situations of violence experienced by students in the state capitals and the Federal District: results from the National Adolescent School-based Health Survey (PeNSE 2012). Revista Brasileira de Epidemiologia. 2014;17:s158-171.

44. Tonkin RS, Murphy A, Chittenden M, et al. Health Youth Development: Highlights from the 2003 Adolescent Health Survey. [Internet]. Vancouver (BC): McCreary Centre Society; 2004. En ligne à : http://www.mcs.bc.ca /pdf/AHS-3_provincial.pdf

45. Tonkin RS. British Columbia Youth Health Trends: A Retrospective, 19922003. [Internet]. Vancouver (BC): McCreary Centre Society; 2005. En ligne à : http://www.mcs.bc.ca/pdf /AHS-Trends-2005-report.pdf

46. Saewyc E, Wang N, Chittenden M, Murphy A. Building resilience in vulnerable youth. [Internet]. Vancouver (BC): McCreary Center Society; 2006. En ligne à : http://www.mcs.bc.ca /pdf/vulnerable_youth_report.pdf

47. Saewyc E, Green R. Survey Methodology for the 2008 BC Adolescent Health Survey IV. Vancouver (BC): McCreary Center Society; 2009.

48. Lau JTF, Kim JH, Tsui HY, Phil M, Cheung A, Lau M et al. The relationship between physical maltreatment and substance use among adolescents: a survey of 95, 788 adolescents in Hong Kong. J Adolesc Health. 2005; 37:110-119. doi: 10.2471/BLT.14.141970.

49. Tang CS. Corporal punishment and physical maltreatment against children: a community study on Chinese parents in Hong Kong. Child Abuse Negl. 2006;30:893-907.
50. Ajdukovic M, Susac, N, Rajter $M$. Gender and age differences in prevalence and incidence of child abuse in Croatia. Croat Med J. 2013;53:469-479. doi: $10.3325 / \mathrm{cmj} .2013 .54 .469$.

51. Helweg-Larsen K, Frederiksen ML, Larsen HB. Violence, a risk factor for poor mental health in adolescence: a Danish nationally representative youth survey. Scand J Public Health. 2011; 39(8):849-856. doi: 10.1177/14034948 11421638 .

52. Frederiksen ML, Helweg-Larsen K, Larsen HB. Self-reported violence amongst adolescents in Denmark: is alcohol a serious risk factor? Acta Paediatr. 2008;97(5):636-640. doi: 10.1111/j.1651-2227.2008.00735.x.

53. Van Gastel WA, Tempelaar W, Bun C, Schubart CD, Kahn RS, Plevier C, et al. Cannabis use as an indicator of risk for mental health problems in adolescents: a population-based study at secondary schools. Psychol Med. 2013; 43(9):1849-1856. doi: 10.1111/j.1651 -2227.2008.00735.x.

54. Sariola H, Uutela A. The prevalence and context of family violence against children in Finland. Child Abuse Negl. 1992;16(6):823-832.

55. Ohene SA, Johnson K, AtunahJay S, Owusu A, Borowsky IW. Sexual and physical violence victimization among senior high school students in Ghana: risk and protective factors. Soc Sci Med. 2015;146:266-275. doi: 10.1016/j .socscimed.2015.10.019.

56. World Health Organization. Global School-Based Student Health Survey (GSHS) Purpose and Methodology [Internet]. En ligne à : http://www .who.int/chp/gshs/methodology/en/

57. Fotiou A, Kanavou E, Richardson C, Ploumpidis D, Kokkevi A. Misuse of prescription opioid analgesics among adolescents in Greece: the importance of peer use and past prescriptions. Drugs: Educ Prev Polic. 2014;21(5): 357-369. doi: 10.3109/09687637.2014 .899989.
58. Flynn-O’Brien KT, Rivara FP, Weiss NS, et al. Prevalence of physical violence against children in Haiti: a national population-based cross sectional survey. Child Abuse Negl. 2016;51:154-162. doi: 10.1016/j.chiabu .2015 .10 .021 .

59. Patel V, Andrew G. Gender, sexual abuse and risk behaviours in adolescents: a cross-sectional survey in schools in Goa. Natl Med J India. 2001;14(5):263-267.

60. Mahram M, Hosseinkhani Z, Nedjat S, Aflatouni A. Epidemiologic evaluation of child abuse and neglect in schoolaged children of Qazvin province, Iran. Iran J Pediatr. 2013;23(2): 159-164.

61. Okech JEA. A multidimensional assessment of children in conflictual contexts: the case of Kenya. Int J Adv Couns. 2012;34(4):331-348.

62. Frias SM, Erviti J. Gendered experiences of sexual abuse of teenagers and children in Mexico. Child Abuse Negl. 2014;38(4):776-787. doi: 10.1016/j .chiabu.2013.12.001.

63. Ahmad N, Cheong SiewMan, Nurashikin Ibrahim, Azriman R. Suicidal ideation among Malaysian adolescents. (Supplement Issue: Malaysian school-based survey 2012.). Asia-Pac J Public Health. 2014;26(5): 63S-69S.

64. Ahmed A, Wan-Yen C, Marret MJ, et al. Child maltreatment experience among primary school children: A large scale survey in Selangor state, Malaysia. PLoS ONE. 2015;10(3).

65. Klein K, Boersma AA, Meyboomde Jong B, de Bruijn J. Child abuse: a common problem in Curacao? West Indian Med J. 2013;62(2):127-134.

66. Denny SJ, Grant S, Utter J, Robinson EM, Fleming TM, Milfont TL, et al. Health and well-being of young people who attend secondary school in Aotearoa, New Zealand: What has changed from 2001 to 2007? J Paediatr Child Health. 2011;47(4):191-197. doi: 10.1111/j.1440-1754.2010.01945.x. 
67. Fleming $\mathrm{T}$, Watson $\mathrm{P}$, Robinson $\mathrm{E}$, Ameratunga S, Dixon R, Clark T, et al. Violence and New Zealand young people: findings of Youth 2000 - A National Secondary School Youth Health and Wellbeing Survey. Auckland (New Zealand): The University of Auckland; 2007.

68. Adolescent Health Research Group. Youth'07: The Health and Wellbeing of Secondary Schools Students in New Zealand. Auckland (New Zealand): The University of Auckland; 2008.

69. Fry D, Anderson J, Hidalgo RJT, Elizalde A, Casey T, Rodriguez R, et al. Prevalence of violence in childhood and adolescence and the impact on educational outcomes: evidence from the 2013 Peruvian national survey on social relations. Int Health. 2016;8(1): 44-52.

70. Al-Quaiz AM, Raheel HM. Correlates of sexual violence among adolescent female in Riyadh, Saudi Arabia. Saudi Med J. 2009;30(6):829-834.

71. Al-Eissa MA, Saleheen HN, Almadani $S$, et al. Determining prevalence of maltreatment among children in the kingdom of Saudi Arabia. Child Care Health Dev. 2016;42(4):565-571.

72. International Society for the Prevention of Child Abuse and Neglect (ISPCAN). Outil de dépistage de la violence des enfants (ICAST-CH) [Internet]. [consultation le 7 octobre 2016]. En ligne à : http://www.ispcan .org/page/ICAST.

73. Rajindrajith S, Devanarayana NM, Lakmini C, et al. Association between child maltreatment and constipation: a school-based survey using Rome III criteria. J Pediatr Gastroenterol Nutr. 2014;58(4):486-490. doi: 10.1097/MPG .0000000000000249 .

74. Andersson N, Ho-Foster A. 13,915 reasons for equity in sexual offences legislation: a national school-based survey in South Africa. Int J Equity Health. 2008;7:20.

75. Waller R, Gardner F, Cluver L. Shared and unique predictors of antisocial and substance use behavior among a nationally representative sample of South African youth. AggressViolent Behav. 2014;19(6):629-636. doi: 10.1016 /j.avb.2014.09.002.
76. Young Carers Project South Africa. Teen Talk South Africa [Internet]. [consultation le 16 octobre 2016]. En ligne à : https://static1.squarespace.com /static/54e3c4b3e4b02a415877e452 /t / 55 f 2 e $1 \mathrm{ffe} 4 \mathrm{~b} 04671$ a 3 ecb $62 \mathrm{~b}$ /1441980927196/Teen + Talk + $1+$ -ENGLISH-27-Jan-20101.pdf

77. Mossige S, Ainsaar M, Svedin CG. The Baltic Sea Regional Study on Adolescent Sexuality. Oslo: Norwegian Social Research (NOVA); 2007.

78. Yen CF, Yang MS, Yang MJ, et al. Childhood physical and sexual abuse: prevalence and correlates among adolescents living in rural Taiwan. Child Abuse Negl. 2008;32:429-438. doi: 10.1016/j.chiabu.2007.06.003.

79. Sofuglu Z, Oral R, Aydin F, et al. Epidemiological study of negative childhood experiences in three provinces of Turkey. Türk Ped Ars. 2014;49:47-56. doi: 10.5152/tpa.2014.838.

80. Jackson V, Browne K, Joseph S. The prevalence of childhood victimization experienced outside of the family: Findings from an English prevalence study. Child Abuse Negl. 2016;51:343357. doi: 10.1016/j.chiabu.2015.08.006

81. Radford L, Corral S, Bradley C, Fisher H. The prevalence and impact of child maltreatment and other types of victimisation in the UK: Findings from a population survey of caregivers, children and young people and young adults. Child Abuse Negl. 2013;37: 801-813.

82. Finkelhor D, Omrod R, Turner $H$, Hamby S. School, police, and medical authority involvement with children who have experienced victimization. Arch Pediat Adol Med. 2011;165(1):9-15. doi: 10.1001/archpediatrics.2010.240.

83. Mitchell KJ, Finkelhor D, Wolak J, Ybarra ML, Turner H. Youth internet victimization in a broader victimization context. J Adolesc Health. 2011;48(2): 128-134. doi: 10.1016/j.jadohealth.2010 .06 .009

84. Finkelhor D, Hamby SL, Omrod R, Turner $\mathrm{H}$. The Juvenile Victimisation Questionnaire: reliability, validity and national norms. Child Abuse Negl. 2005;29(4):383-412. doi: 10.1016/j. chiabu.2004.11.001.
85. Hamby S, Finkelhor D, Turner $H$. Perpetrator and victim gender patterns for 21 forms of youth victimization in the National Survey of Children's Exposure to Violence. Violence Vict. 2013;28(6):915-939.

86. Hamby SL, Finkelhor D, Ormrod RK, Turner HA. The Juvenile Victimization Questionnaire (JVQ) Administration and Scoring Manual. Durham (NH): Crimes Against Children Research Center; 2005.

87. Finkelhor D, Vanderminden J, Turner H, Hamby S, Shattuck A. Child maltreatment rates assessed in a national household survey of caregivers and youth. Child Abuse Negl. 2014;38(2): 1421-35.

88. Finkelhor D, Turner HA, Shattuck A, Hamby SL. Prevalence of childhood exposure to violence, crime, and abuse: results from the National Survey of Children's Exposure to Violence. JAMA Pediatr. 2015;169(8): 746-754. doi: 10.1001/jamapediatrics .2015 .0676 .

89. Finkelhor D, Shattuck A, Turner H, Hamby S. A revised inventory of Adverse Childhood Experiences. Child Abuse Negl. 2015;48:13-21. doi: 10.1016/j.chiabu.2015.07.011.

90. Strauss MA. Measuring intrafamily conflict and violence: the Conflict Tactics (CT) Scales. J Marriage Fam. 1979;41(1):75-86. doi: 10.2307/351733.

91. McLaughlin KA, Koenen KC, Hill ED, Petukhova M, Sampson NA, Zaslavsky AM. Trauma exposure and posttraumatic stress disorder in a national sample of adolescents. J Am Acad Child Adolesc Psychiatry. 2013;52(8):815830.e14. doi: 10.1016/j.jaac.2013.05.011.

92. McChesney GC, Adamson G, Shevlin M. A latent class analysis of trauma based on a nationally representative sample of US adolescents. Soc Psychiatry Psychiatr Epidemiol. 2015; 50(8):1207-1217. doi: 10.1007/s00127 $-015-1075-5$.

93. Merikangas KR, Avenevoli, S, Costello J, Kortez, D, Kessler RC. The National comorbidity survey adolescent supplement (NCS-A): 1 Background and measures. J Am Acad Child Adolesc Psychiatry. 2009;48(4):367-369. doi: 10.1097/CHI.0b013e31819996f1. 
94. Danielson CK, Macdonald A, Amstadter $\mathrm{AB}$, Hanson RF, de Arellano MA, Saunders BE, Kilpatrick DG. Risky behaviors and depression in conjunction with-or in the absence of-lifetime history of PTSD among sexually abused adolescents. Child Maltreat. 2010;15(1): 101-107. doi: 10.1177/1077559509350075.

95. Hawkins AO, Danielson CK, de Arellano MA, Hanson RF, Ruggiero KJ, Smith DW, et al. Ethnic/racial differences in the prevalence of injurious spanking and other child physical abuse in a national survey of adolescents. Child Maltreat. 2010;15(3):242249. doi: 10.1177/1077559510367938.

96. McCauley JL, Danielson CK, Amstadter $\mathrm{AB}$, Ruggiero KJ, Resnick HS, Hanson $\mathrm{RF}$, et al. The role of traumatic event history in non-medical use of prescription drugs among a nationally representative sample of US adolescents. J Child Psychol Psyc. 2010;51(1):84-93. doi: 10.1111/j.1469-7610.2009.02134.x.

97. McCart MR, Zajac K, Danielson CK, Strachan M, Ruggiero KJ, Smith DW, et al. Interpersonal victimization, posttraumatic stress disorder, and change in adolescent substance use prevalence over a ten-year period. J Clin Child Adolesc Psyc. 2011;40(1):136143. doi: 10.1080/15374416.2011.533411.

98. Andrews AR3rd, Jobe-Shields L, Lopez CM, Metzger IW, de Arellano MAR, Saunders B, et al. Polyvictimization, income, and ethnic differences in trauma-related mental health during adolescence. Soc Psychiatry Psychiatr Epidemiol. 2015;50(8):1223-1234. doi: 10.1007/s00127-015-1077-3.

99. Haley CC, Hedberg K, Leman RF. Disordered eating and unhealthy weight loss practices: which adolescents are at highest risk? J Adolesc Health. 2010;47(1):102-105.

100. Oregon Public Health Division. 2005 results. Oregon Healthy Teen Survey [Internet]. Portland (OR): Oregon Health Authority; 2005 [consultation le 5 mai 2016]. En ligne à : https://public.health .oregon.gov/BirthDeathCertificates /Surveys/OregonHealthyTeens/2005 /Pages/index.aspx
101. Alriksson-Schmidt AI, Armour BS, Thibadeau JK. Are adolescent girls with a physical disability at increased risk for sexual violence? J Sch Health. 2010;80(7):361-367. doi: 10.1111/j.1746 $-1561.2010 .00514 . x$.

102. Eaton DK, Kann L, Kinchen S, Ross J, Hawkins J, Harris WA, et al. Methodology of the youth risk behaviour surveillance - United States, 2005. MMWR Surveill Sum. 2006;55:1-108.

103. Brener ND, Kann L, Kinchen SA, Grunbaum JA, Whalen L, Eaton D, et al. Methodology of the youth risk behavior surveillance system. MMWR Recomm Rep. 2004;53(RR12):1-14.

104. Howard DE, Wang MQW. Psychosocial correlates of U.S. adolescents who report a history of forced sexual intercourse. J Adolesc Health. 2005;36(5): 372-379. doi: 10.1016/j.jadohealth 2004.07.007.

105. Lippe J, Brener N, Kann L, Kinchen S, Harris WA, McManus T, et al. Youth risk behaviour surveillance - Pacific Island United States territories, 2007. MMWR Surveill Summ. 2008;57(12): 28-56.

106. Peleg-Oren N, Cardenas GA, Comerford M, Galea S. Exploratory study on the association between interpersonal violence experiences and alcohol use among adolescents. Soc Work Res Sep. 2013;37(3):277-285. doi: 10.1093 /swr/svt016.

107. Brown DW, Riley L, Butchart A, Meddings DR, Kann L, Harvey AP. Exposure to physical and sexual violence and adverse health behaviours in African children: results from the Global School-based Student Health Survey. Bull World Health Organ. 2009;87(6):447-455. doi: 10.2471/BLT .07 .047423 .

108. Kassis W, Puhe H. Data Protection and Code of Research Ethics. Osnabruck (Allemagne): University of Osnabruck; 2009.

109. Durant J, Ensom R. Physical punishment of children: lessons from 20 years of researchCan Med Assoc J. 2012;184(12):1373-1377. doi: 10.1503 /cmaj.101314.
110. Clément ME, Berube A, Chamberland C. Prevalence and risk factor of child neglect in the general population. Public Health. 2016;138:86-92. doi: 10.1016/j.puhe.2016.03.018

111. Kaufman Kantor G, Holt MK, Mebert CJ, Straus MA, Drach KM, Ricci LR, et al. Development and preliminary psychometric properties of the multidimensional neglectful behavior scale child report. Child Maltreat. 2004; 9(5):409-428. doi: 10.1177/10775595 04269530 .

112. Turner RJ, Wheaton B. Checklist measurement of stressful life event. In: Cihen S, Kessler RC, Underwood GL, editors. Measuring stress: a guide for health and social scientists. Oxford University Press; 1997:29-53.

113. Arata CM, Langhinrichsen-Rohling J, Bowers D, O’Brien N. Differential correlates of multi-type maltreatment among urban youth. Child Abuse Negl. 2007;31(4):393-415.

114. Ybarra ML, Langhinrichsen J, Friend J, Dienner-West M. Impact of asking sensitive questions about violence to children and adolescents. J Adolesc Health. 2009;45:499-507. doi: 10.1016 /j.jadohealth.2009.03.009.

115. Langhinrichsen-Rohling J, Arata C, O’Brien N, Bowers D, Klibert, J. Sensitive research with adolescents: just how upsetting are self-report surveys anyways? Violence and Victims 2006;21(4):425-444

116. Finkelhor D, Vanderminden J, Turner H, Hamby S, Shattuck A. Upset among youth in response to questions about exposure to violence, sexual assault and family maltreatment. Child Abuse Negl. 2014;38(2):217-223. doi: 10.1016 /j.chiabu.2013.07.021.

117. Zajac L, Ruggieor KJ, Smith DW, Saunders BE., Kilpatrick DG. Adolescent distress in traumatic stress research: Data from the National Survey of Adolescents-Replications. J Trauma Stress. 2011;24(2):226-229. doi: $10.1002 /$ jts.20621. 
118. DeMarni Cromer L, Freyd JJ, Binder AK, De Prince AP, Becker-Blease K. What's the risk in asking? Participants' reaction to trauma history questions compared with reaction to other personal questions. Ethics Behav. 2006; 16:347-362. doi: 10.1207/s15327019 eb1604_5.

119. Hovdestad W, Tonmyr L. Proposal of childhood maltreatment questions for inclusion on a Canadian national health survey: notes from the process. Dans Gray J (dir.), World Perspectives on Child Abuse, 9e éd. International Society for the Prevention of Child Abuse and Neglect (ISPCAN); 2010. p. 43-48.

120. Tonmyr L, Hovdestad W. Public health approach to child maltreatment. J Paediatr Child Health. 2013;18(8): 411-413. 